\title{
Colangiocarcinoma en un paciente con enfermedad de Wilson
}

\author{
Cholangiocarcinoma in a patient with Wilson's disease
}

\author{
Fernando Rocha Correia, Rogério Corga da Silva, João Andrade, Alfredo Pinto \\ ULSAM. Portugal
}

\begin{abstract}
The incidence of hepatobiliary malignancies, and especially intrahepatic cholangiocarcinoma (ICC), for patients with Wilson's disease (WD), is very low, even for cirrhotic patients. It has been suggested that hepatobiliary carcinomas are less frequent in Wilson's disease (WD) than in liver diseases of other etiology. However, the protective role of copper against malignancies is debated. Only a few cases of cholangiocarcinoma (CCC) in WD have been published. Here we report a case of a 39-year-old female, WD diagnosed at 19-year-old based on low ceruloplasmin level, low serum copper, and increased urinary copper. She was followed up and treated with chelating agents throughout nineteen years, after this asymptomatic period she reported abdominal pain. MRI showed hepatic lesions not suggestive of hemangiomas and a nodular lesion of $15 \mathrm{~mm}$ in the right lobe. Liver biopsy was made, with non-specific fibrosis; negative for malignancy. Few months later she was admitted in the hospital because of rapidly development of epigastric/right hypochondrium abdominal pain with four days of evolution, anorexia and asthenia. Biopsy revealed cholangiocarcinoma as the primary tumor confirmed by strong CK7 and CK20 positivity. The curiosity of the presented case is the very rapid development of CCC despite continuous chelating agent therapy.
\end{abstract}

Palabras clave: Enfermedad de Wilson; Cobre; Colangiocarcioma.

Keywords: Wilson's disease; Copper; Cholangiocarcinoma.

\section{INTRODUCTION}

Wilson's Disease (WD) was first described in 1912 when doctor Kinnier Wilson published the article "Progressive Lenticular Degeneration: A Familial Nervous Disease Associated with Cirrhosis of the Liver" in the "BRAIN" medical journal'. Since then, there were various studies that had in common the difficulty in gathering a significant and representative sample of patients, to allow a good quality and powerful evaluation, and/ or randomization to study therapies and strategies.

WD is a rare genetic condition known to be caused by a mutation in the ATP7B gene (the also known as "Wilson gene"), that is hereditary in a recessive form, and results in a defective biliary elimination of Copper with the consequent toxic accumulation in different organs. This gene encodes a protein (ATPase) with the main function in the transmembrane transport of cooper in the liver (from out of the hepatocytes to the bile). When this shift is compromised by the genetic defect, the cellular accumulation leads to inflammation, and subsequent passage to blood stream, with the deposition in other organs (such as brain and kidneys), leading to its correspondent toxicity. The loss of this protein function is also responsible for the ceruloplasmine failure to uptake cooper. There are more de 500 mutations described in the Wilson gene, and the incidence of this condition is $1: 30000^{2}$. Although it could appear at any age, it is more usual to be diagnosed between 5 and 35 years. Its diagnosis is greatly dependent on medical suspicion and direct testing. In cases of neurologic symptoms (ataxia, rigid-akinetic syndromes, cognitive deterioration, or psychiatric symptoms), the diagnosis seems to be quicker and straightforward. The clinical manifestations are very heterogeneous, depending on the organ/organs affected, and the degree of the lesion. Ideally, patients should be identified and diagnosed before they achieved advanced liver damage with neurological symptons and Kayser-Fleischer rings. The liver damage might precede neurological symptoms in years, but typically, most of the neurological patients will have hepatic changes by the time of diagnosis. Signs related to liver damage are variable, since asymptomatic analytic findings (typically the augmented bilirubin with normal or abnormally low alkaline phosphatase and slightly elevated aminotransferases), to the other edge of the spectrum, with established cirrhosis accompanied by portal hypertension, or an acute liver failure. It is important to point out that the Wilson gene can give origin to any type of liver disease and/or neurological- psychiatric symptoms or Coombs negative hemolytic anemia ${ }^{3}$. One of the most typical and famous findings are already mentioned Kayser-Fleischer rings, found in the cornea, but they're neither specific nor a sensible finding of WD.

One growingly question relates to the oncologic complications of WD. Being a cause of chronic liver disease and cirrhosis, it is a known risk for Hepatocellular carcinoma (HCC), it has fallen under the usual cirrhotic lesion screening program. It has been suggested that HCC seems to be less frequent in WD than in liver diseases of other origin ${ }^{4,5}$. Actually, some studies have shown that the cases of malignancy in WD are rare, even when cirrhosis is established ${ }^{6}$, when compared to cirrhosis from other causes. In this sense, some authors defend a protective role of copper against malignancies, describing an effect in nuclear chromatin stabilization, and studies in rats showed that an excess in copper intake protect against chemically induced carcinogenesis? . On the other hand, an augmented amount of Copper induces DNA damage? 
If HCC in WD patients is difficult to analyze, as a consequence of its low incidence, the evaluation is even more difficult for the even rarer cholangiocarcinoma (CCC) in WD. Here we report on a case with rapidly developed CCC in a 39-year-old female WD patient who had been followed up and treated with chelating agents throughout nineteen years.

\section{CASE REPORT}

This Caucasian female patient was sent to internal medicine consultation at the age of 19 years for presenting jaundice and elevation of transaminases. She was asymptomatic and there were no risk factors for hepatic disease (intravenous drug use, accidental needle punctures or unknown origin cuts, unprotected sexual contacts, alcohol or natural herbal products consumption, and no blood transfusions).

On the objective examination there were no stigmas of chronic liver disease. No hepatomegaly or splenomegaly, and no signs of portal hypertension (no semiology of ascites, and neither visible collateral venous circulation, nor edema). Laboratory tests showed a moderate transaminase elevation (AST 50 IU/L, ALT $96 \mathrm{IU} / \mathrm{L}$ ) and alkaline phosphatase (158 IU/L), with normal gamma-GT (272 IU/L) levels. Serum bilirubin and blood counts were normal. The Anti-mitochondrial and antismooth muscle antibodies were negative. Serologic evaluation of hepatitis $B$, hepatitis $C$ and HIV were negative. Serum copper $(33 \mu \mathrm{g} / \mathrm{dL})$ and ceruloplasmin $(7 \mathrm{mg} / \mathrm{dL})$ levels were both decreased.

Liver biopsy was performed and showed hepatic parenchyma and slightly fibrous portal spaces with inflammatory infiltrate of mononuclear cells. Hepatocytes with focal steatosis, concluding as chronic active hepatitis.

The proper diagnosis of WD was established two years thereafter, based on low ceruloplasmin level $(<7 \mathrm{mg} / \mathrm{dL})$, low serum copper $(27 \mu \mathrm{g} / \mathrm{dL})$ and increased urinary copper (153 $\mu \mathrm{g} / 24 \mathrm{~h})$. No Kayser-Fleischer rings were seen. Serial abdominal ultrasound showed no abnormalities. The patient remained asymptomatic. She started treatment with D-Penicillamine, presenting a rash that led to his suspension. No proteinuria or neutropenia was observed.

A new treatment was started with Trientine Hydrochloride. There was a subsequent normalization of the transaminases and urinary copper levels remained within the desired range. There was a need for dose reduction during pregnancy, resuming four tablets/day after delivery.

She remained asymptomatic and with normal liver tests due to chelating therapy for seventeen years. The regular ultrasound monitoring did not identify focal liver lesions. Seventeen years later, she returned to the consultation with abdominal pain referred to the right hypochondrium. A new ultrasound study was performed, demonstrating a structural alteration of the liver and a lacunar lesion of about $3 \mathrm{~cm}$. To better characterize the identified lesion, abdominal CT was performed, and the lesion was described as being suggestive of hemangioma.

Six months later, a new ultrasound scan was executed, excluding focal lesions and, at the request of the family doctor, she performed a new CT scan that also excluded focal lesions.
After another six months period, she was reevaluated with an abdominal ultrasound that showed an hyperechogenic and heterogeneous images in segment III $(3.7 \mathrm{~cm} / 1.7 \mathrm{~cm} / 3.5 \mathrm{~cm})$. Blood counts, alpha-fetoprotein, AST, ALT, GGT and alkaline phosphatase were normal. She performed an abdominal MRI that showed hepatic lesions that were not suggestive of hemangiomas, the most voluminous occupying almost the entire left lobe, associated with left portal vein thrombosis. Nodular lesion of $15 \mathrm{~mm}$ in the right lobe. The left lobe neoformation was biopsied, with a description of "non-specific fibrosis, negative for malignancy. Parenchyma without neoformation; steatosis and very slight portal inflammation". The patient was referred to a consultation at the Liver Transplatation Reference Center of our Hospital due to hepatic infarction following portal thrombosis and nodule in the right lobe to be clarified.

A few months later, at the age of 39-years old, she was admitted at the hospital with symptoms of rapidly aggravating abdominal pain in the epigastric/right hypochondrium, with four days of evolution, accompanied by anorexia and asthenia. The following complementary diagnostic tools were performed: Serum bilirubin and blood counts were normal. The tumor markers AFP and CEA were in normal range, but CA125 level was very high $(675,8 \mathrm{U} / \mathrm{ml})$.

Abdominal ultrasound: mild ascites. Hepatic dysmorphism, left lobe atrophy and compensatory hypertrophy of the right lobe. In the left lobe there was a massive hypoechogenic solid formation, previously described, of similar volume. Appearance of at least 3 solid, hypoechogenic nodular images in the right lobe with 38,32 and $17 \mathrm{~mm}$, apparently of malignancy origin. Pelvic MRI: Multiple peritoneal implants in the pelvis, more evident in the Douglas bottom and in the left adnexal region, compatible with metastatic implants. Ovaries without lesions suspected of malignancy. No changes in the uterus or endometrium. Abdominal MRI: Predominantly hyperintense area occupying almost all of the left hepatic lobe. Significant increase in nodule size already identified in segment IV $(4 \mathrm{~cm})$. A second node in segment $V$ with $2.5 \mathrm{~cm}$. In the hepatic peri-hilar region, a nodular conglomerate, probably translating adenomegaly agglomerate or even secondary neoplastic implant. Large volume ascites, with multiple peritoneal nodules being identified, and secondary neoplastic implants are likely to be present. Multiple peri-celiac adenomegalies. Hepatic Nodule Biopsy: Morphological findings compatible with hepatic primitive malignant epithelial neoplasia (Cholangiocarcinoma / Hepatocarcinoma). The cellular morphology is identical to that described in the peritoneal. Abdominal biopsy: Morphological findings compatible with malignant epithelial neoplasia. After extensive immunohistochemical study for the marker Hep Par (NEGATIVE) and Keratin (CK7 positive, CK20 positive), we concluded Cholangiocarcinoma.

At the hospital, she stayed hemodynamically stable; maintained ascites in need of evacuation paracentesis. She was oriented to the external consultation of Internal Medicine. She presented a progressive worsening of clinical status with refractory ascites, periods of encephalopathy, anorexia and extreme asthenia. She died a month later. 


\section{DISCUSSION}

First, as previously reported, WD is a particularly rare illness, and this case, simply by this fact of representing an infrequent condition raised our interest since the beginning, especially when we consider that it had been identified and diagnosed early, corresponding to a young adult, and yet under medical supervision, and with apparently correct therapeutic, the outcome still was unfortunate, with the development of a neoplasm and death.

WD is a diagnose that demands a direct suspicion from the medical doctor that is studying the clinical picture. There were no Kayser-Fleischer rings, which are a usual finding in patients with neurological symptoms, but are commonly absent in exclusive hepatic involvement, and the liver biochemical blood tests weren't the typical either (there wasn't the hyperbilirubinemia that usually accompanies the slight elevation of alkaline phosphatase). In this case, the biochemical presentation of the Copper study (urinary copper and ceruloplasmin) was diagnostic and proper treatment (after different options had been tried) did managed to control and achieve the expected and wanted normalization. There were no comments by the pathologist that read the liver biopsy regarding the diagnosis of WD, but the center where this was performed had no resources to measure the Copper content in dry liver. Still, other etiologies were excluded.

With timely identification and treatment being one of the main goals in order to prevent progression to cirrhosis or other complications, this case, identified without cirrhosis established, provided the (soon to be confirmed as false) security of good prognosis. The patient maintained values of serum and urinary copper controlled under Trientine Hydrochloride and cytolysis markers in the normal range. The appearance of a nodular lesion in the routine ultrasound study, promptly lead to alternative diagnostic exams (with a more detailed characterization by abdominal CT and MRI). Initially described as hemangioma, and under surveillance. Subsequent exams showed the oncologic lesion.

The difficulty in diagnosing and studying this kind of complications, is clearly demonstrated in this clinical setting, where the patient actually performed an initial liver biopsy that was negative for malignancy. Finally, the identification of cholangiocarcinoma (with the aid of abdominal metastatic implants), confirmed the medical team worst expectations.

The hepatic neoplasia most often described in the literature as being associated with Wilson's disease is hepatocarcinoma. CCC related to WD has been described in sporadic case reports, and there is no consensus into its relation or the incidence. In this case there was a rapid growth of CCC despite 19 years of effective therapy and regular ultrasound monitoring.

\section{CONCLUSION}

Due to the lack of studies and the existence of conflicting theories affirming both reduced and increased risk of hepatic neoplasia in patients with Wilson's disease, the authors recommend that a preventive approach should be adopted. We consider, and this patient was a particular difficult example, that a preemptive and regular imaging screening should be performed. We agree with the hepatic ultrasound screening, with interval similar to that used in cirrhotic patients (every six month) should be applied, with alternative imaging studies (CT or MR) and shorter intervals (3 months) applied when facing a new and/or undefined nodule. We agree with the common conclusion in the publications related to Wilson disease, that more studies and bigger populations are needed to understand more and better this condition.

Conflicts of interest: the authors declare that there is no potential conflict of interest relevant to this article.

Authors' contribution: Correia, Fernando Rocha and Corga da Silva, Rogério wrote de paper; Andrade, João and Pinto, Alfredo reviewed the paper.

\section{BIBLIOGRAPHY}

1. Wilson SAK. Progressive lenticular degeneration: a familial nervous disease associated with cirrhosis of the liver. Brain. 1912;34:295-507

2. A Review and Current Perspective on Wilson Disease. MallikarjunPatil, Keyur A. Sheth, Adarsh C. Krishnamurthy, and Harshad Devarbhavi J ClinExpHepatol. 2013; 3(4): 321-336.

3. Mapping, cloning and geneticcharacterization of the region containing the Wilson disease gene. Petrukhin K, Fischer SG, Pirastu M, et al. NatGenet. 1993;5:338343

4. No increased risk of hepatocellular carcinoma in cirrhosis due to Wilson disease during longtermfollow-up. van Meer S, de Man RA, van den Berg AP, et al. J Gastroenterol Hepatol. 2015;30:535-539.

5. Long-term outcomes of patients with wilson disease in a large Austrian cohort. Beinhardt S, Leiss W, Stättermayer AF, et al. Clin Gastroenterol Hepatol. 2014;12:683-389.

6. Intrahepatic cholangiocarcinoma in a patient with Wilson's disease: a case report. Yosuke Mukai et al. Surgical Case Reports. 2016; 2:29.

7. Hepatobiliary malignancies in Wilson disease. Pfeiffenberger J, Mogler C, Gotthardt DN, et al. Liver Int. 2015;35:1615-1622. 